\title{
Area-Preserving Mappings for the Visualization of Medical Structures
}

\author{
Lei Zhu ${ }^{1}$, Steven Haker ${ }^{2}$, and Allen Tannenbaum ${ }^{1 \star}$ \\ 1 Department of Biomedical Engineering, \\ Georgia Institute of Technology, Atlanta, GA 30332 \\ gte538w@prism.gatech.edu, tannenba@ece.gatech.edu \\ 2 Surgical Planning Lab, Brigham and Women's Hospital, \\ Harvard Medical School, Boston, MA 02115 \\ haker@bwh. harvard . edu
}

\begin{abstract}
In this note, we present a method for flattening anatomical surfaces such as branched vessels and intestinal tracts in an areapreserving way. This method is based on the theory of optimal mass transport and conformal mapping of surfaces. The flattened representations differ minimally from conformality in a certain precise sense. Potential applications include the detection and visualization of pathologies such as stenoses and polyps.
\end{abstract}

\section{Introduction}

Surface deformations, in particular the flattening of highly undulated and branched surfaces, is an active area of research in the field of medical imaging and visualization, For example, flattened representations of brain surface are important in the study of neural activities within the three dimensional folds of brain surface; see 14,2 and the references therein. Flattened versions of the colon surface may be a good complement for CT colonography or virtual colonoscopy; see 12,7 and the references therein.

There have been a number of approaches to surface flattening. In[12], a visualization technique is proposed which uses cylindrical and planar map projections. Methods based on quasi-isometric and quasi-conformal flattenings of brain surface have been considered in 413], among others. Wang [15] presented a method for unravelling colon surface based on electrical field. Typically, these methods do not guarantee bijectivity of the mapping. Algorithms based on conformal mapping have also been applied on this problem [2716]. These methods preserve angles, and in this sense preserve local geometry. They also guarantee bijectivity. However, these conformal flattening methods are not area-preserving, since a mapping cannot be both angle-preserving and area-preserving unless the original surface has zero Gaussian curvature. This problem becomes particularly

\footnotetext{
* Allen Tannenbaum is also with the School of Electrical and Computer Engineering. This work was partially supported by grants from NSF, ARO, AFOSR, MRI-HEL, and NIH, including NIH R01 AG19513.
} 
pronounced when we wish to construct a flattened representation for a multibranched surface.

Here we take another approach based on the theory of optimal mass transport. Our method makes minimal adjustments to an initial conformal mapping to find an area-preserving mapping. [1].

\section{Conformal Flattening of a Multi-branched Vessel}

We now briefly review our approach for constructing a conformal flattening of a multi-branched vessel. The algorithm contains two steps: dividing the whole vessel into several Y-shaped segments using the harmonic skeleton, and then flattening each Y-shaped segment. More precisely, our method is based on the solution of a harmonic equation on the surface, from which a "harmonic skeleton" or medial axis is derived. The harmonic skeleton is easy to calculate, is guaranteed to be smooth, and can be used as a "center line" for a fly-through.

Let $\Sigma$ be a triangulated surface, without self-intersections, which is topologically a tube with several branches. A branched blood vessel is an example of such a surface. We assume each branch terminates in a boundary loop which we call $\sigma_{i}(i=0 \ldots N)$. The first step in our algorithm is to solve a Dirichlet problem $\Delta u=0$ on the interior of $\Sigma$ with proper boundary conditions. One of the boundaries, $\sigma_{0}$, is selected to be the root. The value of $u$ on the root boundary is assigned to be 0 . Given this 0 contour, there exists a set of triangles whose base vertices are coincident with the 0 (starting) contour and whose apex vertices describe the successive contour. This description can be repeated for $n$ pairs of triangle sets until the successive contour reaches a boundary other than root. The value of $u$ on this boundary is then assigned to be $n$. The equation $\Delta u=0$ is solved using standard finite element techniques [7].

The second step is to build a tree-like structure we call the harmonic skeleton. Using the function $u$ solved for in the first step, we find level sets on the surface, i.e. sets $\{x \mid u(x)=\nu\}$ for values of $\nu$ ranging from 0 to the maximum of $u$. We partition each level set into classes according to connectivity on the surface. The centroid of each class of points corresponds to a point on the harmonic skeleton. By increasing $\nu$ from 0 to its maximum, we can build a structured tree. The locations of the bifurcations along the tree are obvious since it is easy to see where one centroid splits into two.

The third step is to refine the harmonic skeleton by replacing the boundary values used previously in step 1 with new values obtained as the distance along the skeleton from the root boundary to the other boundaries. We again solve the corresponding Dirichlet problem and extract a refined harmonic skeleton. It is easy to cut a branched vessel into several Y-shaped segments with the help of this construction.

Details for the conformal flattening of a Y-shaped vessel (i.e., with only a single branch point) have been presented in [16] to which we refer the interested reader. Essentially, the method involves solving another Dirichlet problem to obtain a harmonic conjugate for $u$. 
It is well known that conformal flattenings are "similarities in the small" in the sense that over small regions, the flattened version differs from the original by a scale factor alone. As a result, the flattened surface retains much of the visual quality of the original. However, conformal mappings do not in general preserve surface area. Indeed, some areas of the surface may be greatly enlarged or reduced. The problem becomes exacerbated when there are a large number of branches. To address this problem, we consider adjusting the conformal mapping in a minimal way so as to correct the distortion of area. By making only these minimal adjustments, we hope to preserve the desirable qualities of the conformal mapping as much as possible.

\section{Introduction Optimal Mass Transport}

\subsection{Background}

Our method for minimally adjusting a conformal mapping is based on the theory of optimal mass transport, also referred to as the Monge-Kantorovich problem. Here we present a review of the basic theory. Let $\Omega_{0}$ and $\Omega_{1}$ be two domains in $\mathbf{R}^{\mathbf{2}}$, having smooth boundaries. On each of these domains we assume we are given a priori a mass density function $\mu_{0}$ and $\mu_{1}$ respectively. We assume that the same total mass is associated with each of these densities.

We will be considering a class of diffeomorphisms $u$ from $\Omega_{0}$ to $\Omega_{1}$ which satisfy the equation

$$
\mu_{0}=|D u| \mu_{1} \circ u \text {. }
$$

Here $D u$ is the matrix of first derivatives of $u$, and $\circ$ represents composition of functions. This is the "Jacobian equation", which is a mass preservation (MP) constraint. For a function satisfying (11) we will write $u \in$ MP. The special case where $\mu_{1}=1$ is of particular interest for the current application. In this case Equation (1) reduces to $\mu_{0}=|D u|$, and we see that this equation is simply a hard constraint on the Jacobian $|D u|$ of $u$, the Jacobian giving the amount of scaling of area that occurs locally under the mapping $u$.

We are interested in finding an MP mapping $u$ which differs minimally from the identity. To this end, we introduce a squared $L^{2}$ Kantorovich-Wasserstein penalty functional on $u \in \mathrm{MP}$, defined as:

$$
M[u]:=\int_{\Omega_{0}}\|u(x)-x\|^{2} \mu_{0}(x) d x
$$

This functional (2) is seen to place a penalty on the distance the map $u$ moves each bit of material, weighted by the material's mass. Hence, the KantorovichWasserstein metric may be thought of as the cost associated with transporting, via $u$, a distribution of material given by $\mu_{0}$. The resulting distribution of material is constrained to be the given density $\mu_{1}$, according to (1).

Theoretical results [35] show that there is a unique minimizer $\tilde{u} \in \mathrm{MP}$ of $M$, and that this minimizer is characterized as being the gradient of a convex function $w$, i.e., $\tilde{u}=\nabla w$. 


\subsection{Finding the Minimizer}

There have been many approaches for finding the solution $\tilde{u}$ to the optimal mass transport problem. In [1]8], a new approach for finding the optimal map was proposed, based on the notion of polar factorization 663. Here we present the main idea behind this work.

Our algorithm for finding $\tilde{u}$ consists of two steps. The first step is to find an initial mapping $u$ from $\Omega_{0}$ to $\Omega_{1}$ satisfying the Jacobian equation (1). The second step is to adjust this mapping iteratively using gradient descent in order to minimize the functional $M$, while constraining $u$ so that it continues to satisfy (1). A method for finding an initial mapping is described below. Here, we derive the appropriate gradient descent equation, considering a more general cost functional $M$ given by

$$
M\left[u^{t}\right]=\int_{\Omega_{0}} \Phi\left(u^{t}(x)-x\right) \mu_{0}(x) d x,
$$

where $\Phi: \mathbf{R}^{\mathbf{2}} \rightarrow \mathbf{R}$ is a positive $C^{1}$ function. The $L^{2}$ Kantorovich-Wasserstein functional (2) corresponds to the case $\Phi(x)=\|x\|^{2}$.

We think of $u$ as a function of time $t$, and introduce the notation $u^{t}$, with $u^{0}$ being the initial mapping. Each such $u^{t}$ can be written as the composition

$$
\begin{aligned}
& u^{t}=u^{0} \circ\left(s^{t}\right)^{-1}, \text { or } \\
& u^{0}=u^{t} \circ s^{t},
\end{aligned}
$$

where $s^{t}$ is a mass preserving mapping from $\Omega_{0}$ to itself satisfying $\mu_{0}=\left|D s^{t}\right| \mu_{0} \circ$ $s^{t}$. The derivative of $s^{t}$ with respect to time is described by a vector field $v^{t}$ on $\Omega_{0}$, i.e.

$$
\frac{\partial s^{t}}{\partial t}=v^{t} \circ s^{t} .
$$

where the vector field $v^{t}$ must satisfy $\operatorname{div}\left(\mu_{0} v^{t}\right)=0$ in order to preserve the density $\mu_{0}$. By the chain rule applied to Equation (4), the evolution of $u^{t}$ must satisfy

$$
\frac{\partial u^{t}}{\partial t}+D u^{t} \cdot v^{t}=0
$$

By a change of variables $x=s^{t}(y)$ in (3), together with (4), we find

$$
M(t)=\int_{\Omega} \Phi\left(u^{0}(y)-s^{t}(y)\right) \mu_{0}(y) d y .
$$

From this one computes that the cost decreases according to

$$
\frac{d M}{d t}=-\int\left\langle\nabla \Phi\left(u^{0}-s\right), \frac{\partial s^{t}}{\partial t}\right\rangle \mu_{0} d y=-\int\left\langle\nabla \Phi\left(u^{t}(x)-x\right), \mu_{0} v^{t}\right\rangle d x .
$$

Clearly, were it not for the constraint $\operatorname{div}\left(\mu_{0} v^{t}\right)=0$, we could take $\mu_{0} v^{t}=$ $\nabla \Phi\left(u^{t}(x)-x\right)$ to decrease $M$. Instead, we take $\mu_{0} v^{t}$ to be the divergence-free part 
of $\nabla \Phi\left(u^{t}(x)-x\right)$, finding this part through the use of Helmholtz decomposition. This step involves solving Laplace's equation with boundary conditions set to keep the flow of $s^{t}$ confined in $\Omega_{0}$. The resulting equation for updating $u^{t}$ has the following form:

$$
\frac{\partial u^{t}}{\partial t}=-\frac{1}{\mu_{0}} D u^{t} \cdot\left(\mathrm{I}-\nabla \Delta^{-1} \operatorname{div}\right) \nabla \Phi\left(u^{t}-\underline{\mathrm{id}}\right),
$$

where $\underline{\mathrm{id}}$ denotes the identity map, and I is the identity matrix. For more details on this technique, please refer to [18].

\subsection{Finding an Initial MP Mapping}

A general method for finding an initial mapping for irregularly shaped domains can be found in the work of Moser [10]. In our algorithm, both source and target domains are rectangles of the same size. Accordingly, we can use the following simple method, as described in 18 .

Assume $\Omega_{0}=[0, A] \times[0, B]$ and $\Omega_{1}=[0, A] \times[0, B]$. Our initial mapping will be of the form $u^{0}(x, y)=(a(x), b(x, y))$. Since both $\mu_{0}$ and $\mu_{1}$ are positive, we may define $a(x)$ implicitly by the following equation:

$$
\int_{0}^{a(x)} \int_{0}^{B} \mu_{1}(\eta, y) d y d \eta=\int_{0}^{x} \int_{0}^{B} \mu_{0}(\eta, y) d y d \eta .
$$

Note that $a(0)=0$ and $a(A)=A$ by the assumption that both domains contain same amount of mass. By taking the derivative respect to $x$, we find

$$
a^{\prime}(x) \int_{0}^{B} \mu_{1}(a(x), y) d y=\int_{0}^{B} \mu_{0}(x, y) d y .
$$

We may now therefore define $b=b(x, y)$ implicitly by the equation

$$
a^{\prime}(x) \int_{0}^{b(x, y)} \mu_{1}(a(x), \rho) d \rho=\int_{0}^{y} \mu_{0}(x, \rho) d \rho .
$$

Taking the derivative with respect to $y$ shows that $u^{0}$ satisfies the constraint (11). This construction of $u^{0}$ can be interpreted as finding the optimal mass transport in the $x$ direction, then in the $y$ direction for each $x$.

\section{Optimal Transport Applied to Conformal Mapping}

Our goal is to apply the optimal transport theory to adjust a conformal mapping minimally in order to obtain an area preserving mapping. Let us now assume we have constructed a conformal mapping $f$ of a triangulated surface to a region range $(f)$ in the plane.

For simplicity, and without loss of generality, we assume the initial surface and range $(f)$ have areas equal to 1 . We can always scale homothetically to make 
this so. Define $\mu_{0}$ on range $(f)$ to be the area of a triangle on the original surface divided by the the area of the triangle once flattened. Extend the function $\mu_{0}$ to a rectangular region $\Omega_{0}$ surrounding this range by setting $\mu_{0}$ to 1 outside of range $(f)$. Figure 4 shows such a function $\mu_{0}$, the dark color representing enlarged areas and the light color representing reduced areas. Let $\Omega_{1}=\Omega_{0}$, and set $\mu_{1}$ to be uniformly 1 throughout $\Omega_{1}$.

We now solve the optimal transport problem as described in the previous section. Since $\mu_{1}=1$, the constraint (11) reduces to $\mu_{0}=|D u|$, and so by the construction of $\mu_{0}$ a mapping $u$ which satisfies this constraint compensates exactly for the distortion in area that occurred during flattening. Further, since we minimize the functional (2) to find the minimizing $\tilde{u}$, our solution differs minimally from the identity.

\section{Implementation and Examples}

We use standard techniques to solve Equation (9). In particular we have employed an upwinding scheme when computing $D u^{t}$, and the FFT when inverting the Laplacian on a rectangular grid. Standard centered differences were used for other spatial derivatives. Once we numerically solve for the right hand side of (9), we use the result to update $u^{t}$. The optimal map is obtained as $t \rightarrow \infty$. In practice, we iterate until convergence with respect to a specified tolerance.

Our first example is a dataset provided by the Surgical Planning Lab of Brigham and Women's Hospital, Harvard Medical School. The dataset is a vessel surface extracted from a $256 \times 256 \times 47$ MRA brain image. By using a segmentation method based on active contour model, we found the surface of a section of vessel. We then generated a triangulation of the surface using the Visualization Toolkit as shown in Figure 1 and triangles at the ends were removed to create a tubular surface. Next, we used the technique described in Section 2, we produced a conformal flattening of this surface (Figure 2), together with a harmonic skeleton (Figure 3). Then, as described in Section 4 density maps were generated (Figure 4) based on the distortion of area due to flattening. Figure 5 presents a histogram of this distortion, from which it can be seen that many triangles were enlarged or reduced. Finally, an area-preserving mapping (Figure 6) was constructed based on the algorithm described in Section 3.2 The computation took about 9 minutes for the conformal flattening and about 20 minutes for the area preserve flattening, both on a $1 \mathrm{GHz}$ Linux system. All surfaces in the figures are shaded by using outward normal vectors from the original surface.

The second example is a dataset provided by Emory Hospital. The dataset is a $512 \times 512 \times 91 \mathrm{CT}$ image for carotid vessel. The flattening approach for this dataset was similar to that of the first one, except that there was only one branch point and hence it was not necessary to generate a harmonic skeleton. Figure 7 is the segmented surface and Figure 8 is the area-preserving presentation.

Color images of these examples can be found at: http://www.prism.gatech.edu/ gte538w/miccai2003/miccai2003.htm 


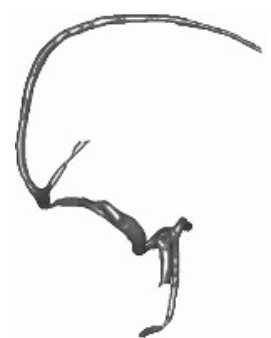

Fig. 1. A segmented brain vessel

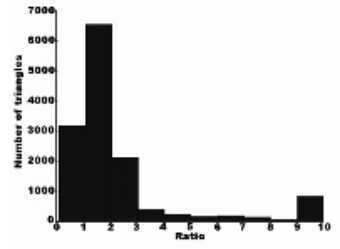

Fig. 5. The statistics for Fig [

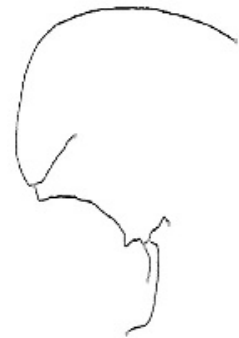

Fig. 3. The harmonic skeleton for Fig 1

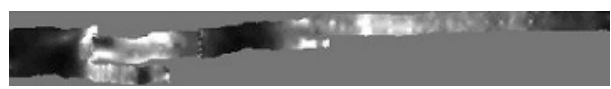

Fig. 4. Density map for Fig 2

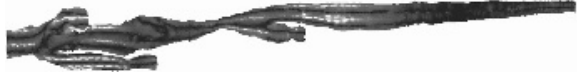

Fig. 6. The area-preserving mapping of Fig. 1

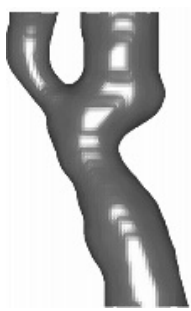

Fig. 7. A segmented carotid vessel

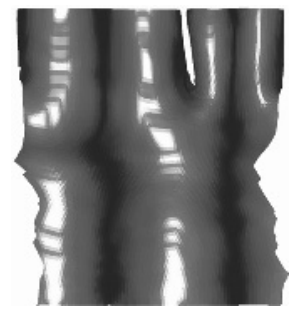

Fig. 8. Area-preserving flattening of Fig 7 


\section{Conclusions}

In this paper, we presented a new method for flattening of branched medical surfaces based on the theory of optimal mass transportation. Starting from a conformal equivalence, we constructed a density map and then generated an areapreserving representation of the original 3D surface. Potential applications include the detection and visualization of pathologies such as stenoses and polyps.

\section{References}

1. S. Angenent, S. Haker, and A. Tannenbaum, "Minimizing flows for the MongeKantorovich problem" to appear in SIAM J. Math. Analysis, 2003.

2. S. Angenent, S. Haker, A. Tannenbaum, and R. Kikinis, "On the Laplace-Beltrami operator and brain surface flattening," IEEE Trans. on Medical Imaging, Vol. 18, pp. 700-711, 1999.

3. Y. Brenier, "Polar factorization and monotone rearrangement of vector-valued functions," Com. Pure Appl. Math. Vol. 64, pp. 375-417, 1991

4. G.J. Carman, H.A. Drury, and D.C. Van Essen, "Computational methods for reconstructing and unfolding the cerebral cortex," Cerb. Cortex, Vol. 5(6), pp. 506-517, 1995

5. W. Gangbo, and R. McCann, "The geometry of optimal transportation," Acta Math. Vol. 177, pp. 113-161, 1996.

6. W. Gangbo, "An elementary proof of the polar factorization of vector-valued functions," Arch. Rational Mechanics Anal. Vol. 128, pp. 381-399, 1994.

7. S. Haker, S. Angenent, A. Tannenbaum, and R. Kikinis, "Nondistorting flattening maps and the 3D visualization of colon CT images," IEEE Trans. on Medical Imaging, Vol. 19, pp. 665-670, 2000.

8. S. Haker and A. Tannenbaum, "Mass-preserving maps for registration and visual tracking" IEEE proceeding on Decision and Control, pp. 4812-4817, 2000.

9. L.V. Kantorovich, "On a problem of Monge", Uspekhi Mat. Nauk, Vol. 3, pp. 225$226,1948$.

10. J. Moser, "On the volume elements on a manifold," Trans. Amer. Math. Soc.Vol. 120, pp. 286-294, 1965.

11. Z. Nehari, Conformal mapping, Dover Publications, New York, 1975.

12. D. Paik, C. Beaulieu, R. Jeffrey, C. Karadi, and S. Napel, "Visualization modes for CT colonography using cylindrical and planar map projections," J. Comput. Assist. Tomogr. Vol. 24(2), pp. 179-188, 2000.

13. E. Schwartz, A. Shaw, and E. Wolfson, "A numerical solution to the generalized mapmaker's problem: flattening noncovex polyhedral surfaces," IEEE Trans. Pattern Anal. Machine Intell. Vol. 11, pp. 1005-1008, 1989.

14. B. Wandell, S. Engel, and H. Hel-Or, "Creating images of the flattened cortical sheet," Invest. Opth. and Vis. Sci. Vol. 36(S612), 1996.

15. G. Wang, S. Dave, B. Brown, Z. Zhang, E. mcFarland, J. Haler, and M.Vannier, "Colon unraveling based on electrical field - Recent progress and further work," SPIE Proc. 3660, 1999.

16. L. Zhu, S. Haker, A. Tannenbaum, S. Bouix and K. Siddiqi, "Angle-preserving mappings for the visualization of multi-branched vessels," in Proc. Image Processing 2002 Vol. 2, pp. 945-948, 2002. 